\title{
Periodic order and defects in Ni-based inverse opal-like crystals on the mesoscopic and atomic scale
}

\author{
A. V. Chumakova, ${ }^{1}$ G. A. Valkovskiy, ${ }^{2, *}$ A. A. Mistonov, ${ }^{1,2}$ V. A. Dyadkin, ${ }^{1,3}$ N. A. Grigoryeva, ${ }^{2}$ N. A. Sapoletova, ${ }^{4}$ \\ K. S. Napolskii, ${ }^{4,5}$ A. A. Eliseev, ${ }^{4}$ A. V. Petukhov,${ }^{6}$ and S. V. Grigoriev ${ }^{1,2}$ \\ ${ }^{1}$ Petersburg Nuclear Physics Institute, Gatchina, 188350 Saint Petersburg, Russia \\ ${ }^{2}$ Faculty of Physics, Saint Petersburg State University, 198504 Saint Petersburg, Russia \\ ${ }^{3}$ Swiss-Norwegian Beamlines at the ESRF, 38000 Grenoble, France \\ ${ }^{4}$ Department of Materials Science, M. V. Lomonosov Moscow State University, 119991 Moscow, Russia \\ ${ }^{5}$ Department of Chemistry, M. V. Lomonosov Moscow State University, 119991 Moscow, Russia \\ ${ }^{6}$ van 't Hoff Laboratory, Debye Institute for Nanomaterials Science, Utrecht University, 3584 CH Utrecht, The Netherlands
}

(Received 5 June 2014; revised manuscript received 13 August 2014; published 13 October 2014)

\begin{abstract}
The structure of inverse opal crystals based on nickel was probed on the mesoscopic and atomic levels by a set of complementary techniques such as scanning electron microscopy and synchrotron microradian and wide-angle diffraction. The microradian diffraction revealed the mesoscopic-scale face-centered-cubic (fcc) ordering of spherical voids in the inverse opal-like structure with unit cell dimension of $750 \pm 10 \mathrm{~nm}$. The diffuse scattering data were used to map defects in the fcc structure as a function of the number of layers in the Ni inverse opal-like structure. The average lateral size of mesoscopic domains is found to be independent of the number of layers. 3D reconstruction of the reciprocal space for the inverse opal crystals with different thickness provided an indirect study of original opal templates in a depth-resolved way. The microstructure and thermal response of the framework of the porous inverse opal crystal was examined using wide-angle powder x-ray diffraction. This artificial porous structure is built from nickel crystallites possessing stacking faults and dislocations peculiar for the nickel thin films.
\end{abstract}

DOI: 10.1103/PhysRevB.90.144103

\section{INTRODUCTION}

Self-assembled colloidal crystals consisting of closepacked spherical particles of submicron size are often referred to as artificial opals. Filling the voids in these opal-like structures with ferromagnetic metals, such as $\mathrm{Ni}, \mathrm{Co}$, and $\mathrm{Fe}$, and removing the colloidal particles allows the fabrication of inverse opals possessing tunable magneto-optical properties [1-7]. An artificial magnetic anisotropy on a mesoscale can also make such inverse opal-like crystals (IOLCs) a unique test bench for the study of unusual magnetic phenomena. Among them, frustrated magnetism and giant magnetoresistance are of special interest, being actively investigated in ordered magnetic micro- and nanostructures in recent years [8-10]. The latest research on magnetic behavior in IOLCs based on cobalt has provided a description of the remagnetization under an applied magnetic field showing that the local configuration of magnetization is inhomogeneous and strongly depends on the IOLC structure [11]. In order to construct the most objective model of magnetization distribution it is important to understand how macroscopic properties arise from the interplay of mesoscopic parameters such as crystal lattice type of IOLC.

The close-packed structure of the self-assembled crystal is formed by stacking hexagonal close-packed layers of colloidal hard spheres at three possible lateral positions A, B, and C such that neighboring layers have different lateral positions (i.e., $\mathrm{AA}, \mathrm{BB}$, and $\mathrm{CC}$ sequences are excluded). A set of three subsequent layers is considered fcc if all of them have different lateral positions (e.g., ABC). Otherwise, it is considered hexagonal close-packed (hcp) if two utmost layers have the same positions (e.g., in ABA). It was predicted theoretically that self-assembled colloidal crystals should

\footnotetext{
*Corresponding author: valkovsky_gleb@mail.ru
}

PACS number(s): 05.65.+b, 61.05.cc, 75.40.-s

favor the fcc structure [12]; i.e., the hexagonal layers are formed in the (111) crystallographic plane and ordered along the [111] crystallographic axis of the artificial opals. In practice, however, a structure with a lot of stacking disorder is commonly realized, which can be regarded in two different ways. One approach is to consider it as a random hexagonal close-packed (rhcp) structure [13-15] being a disordered state intermediate between fcc and hcp. Another interpretation is still to consider such a structure as fcc, while with a considerable amount of stacking faults [16]. Also, our previous works $[5,11,17-20]$ on this issue demonstrated effectiveness of the mesostructure determination using small-angle neutron and synchrotron diffraction.

While much experience has been gained from the interpretation of the diffraction data, there is still no complete description of the nonideality of the diffraction patterns, and, as a consequence, one cannot comprehensively clarify the structural disorder. Moreover, it was shown in [21] that finite-size effects, in particular, surface scattering, should be accounted for in the evaluation of additional scattering features appearing in the diffraction patterns regardless of violation of Bragg's law. In fact, since self-assembled opal-like crystals commonly possess a small number of close-packed hexagonal layers along the growth axis, the thickness effect is likely to be considerable. For instance, when finite-size effects are included, the Bragg peaks are found to be broadened by an amount inversely related to the dimension of the diffraction region of the crystal.

Our present work analyses the Bragg peaks (particularly their broadening) in the small-angle synchrotron diffraction patterns taking into account not only stacking disorder in the growth direction but also finite thickness of the IOLCs. For that a number of IOLCs with different thickness were evaluated. Considering that inverse opals are produced by 
electrochemical deposition, which is supposed to provide highdensity filling of the interstices between the spheres of opal template with metals and very small shrinkage, we suggest an indirect method to depth-resolved-study the layered structure of the original matrices. Still, the detailed nature of the metal walls in IOLCs is to be studied. Indeed, for the comprehensive magnetization evaluation including micromagnetic modeling, it is necessary to take into account not only the mesoscopic but also microstructural properties of the inverse opals.

The paper is organized in the following way. Section II gives the essence of the sample preparation procedure. Section III describes experiments on microradian and wide-angle diffraction carried out at synchrotron radiation facilities. Section IV presents a theoretical introduction to the model used in study of mesoscopic stacking ordering. Sections V and VI consider the respective results of the microradian and wide-angle synchrotron diffraction giving the structural features of IOLCs at different levels. The conclusions and acknowledgments are presented in Secs. VII and VIII, respectively.

\section{SAMPLES}

The IOLC films based on nickel were prepared using a templated electrodeposition. First, colloidal crystal films made of $530 \pm 10 \mathrm{~nm}$ polystyrene microspheres were grown by the electric-field-assisted vertical deposition method onto conducting supports [22,23]. The supports were prepared from single-crystal silicon and mica wafers covered with a 200 $\mathrm{nm}$ thick gold layer. Second, the voids between the spheres were filled with nickel via an electrodeposition technique under potentiostatic mode in three-electrode cell at room temperature. The counterelectrode was a $\mathrm{Pt}$ wire and the reference electrode was a saturated $\mathrm{Ag} / \mathrm{AgCl}$ electrode connected to the cell via a Luggin capillary. Electrocrystallization of $\mathrm{Ni}$ was carried out from an electrolyte containing $0.6 \mathrm{M}$ $\mathrm{NiSO}_{4}, 0.1 \mathrm{M} \mathrm{NiCl}_{2}, 0.3 \mathrm{M} \mathrm{H}_{3} \mathrm{BO}_{3}$, and $3.5 \mathrm{M} \mathrm{C}_{2} \mathrm{H}_{5} \mathrm{OH}$ at a deposition potential of $-0.9 \mathrm{~V}$ versus the reference electrode. Finally, the microspheres were dissolved in toluene to make a freestanding metallic structure on the substrate. A more detailed description of the IOLC film preparation procedure is presented in Ref. [24].

It should be emphasized that the use of the electrochemical technique for metal deposition into the matrix voids enables us to coulometrically control the level of filling and to stop the deposition at a desired depth. Thereby one can obtain a sample with predesigned thickness, which is controlled by in situ measuring of current transients [24] and further checked with cross section scanning electron microscopy (SEM). In particular, for the present study a number of films with gradually increasing thickness were obtained, that is, the samples with $3.5,8,17$, and 26 layers (corresponding to the layers of the initial opal templates). The samples were named $\mathrm{Ni}_{x}$, where $x$ is the number of layers, filled by the metal in the original matrix. The thickness fluctuation of IOLCs within the whole sample area does not exceed $\sim 1$ layer even for the thick samples.

A typical top view SEM image of an IOLC film based on Ni is shown in Fig. 1, and a cross section is presented in the inset in Fig. 1. One can see that metal framework is periodically ordered. As shown in Fig. 1, the direction perpendicular to the substrate typically coincides with the [111] axis of the

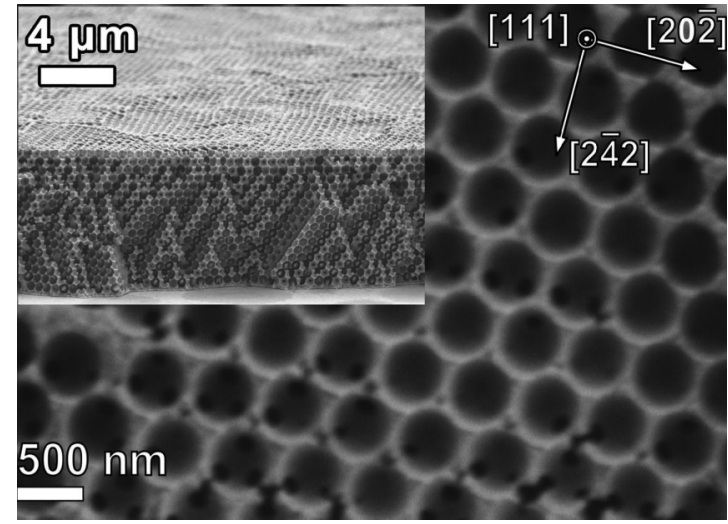

FIG. 1. The typical top view SEM image of IOLC based on Ni. The inset shows the typical cross section SEM image.

fcc structure. Besides, analysis of a number of SEM images allowed us to conclude that the pore walls are built from $\mathrm{Ni}$ crystallites. Their size crudely estimated from the SEM data is $\sim 100 \mathrm{~nm}$.

\section{EXPERIMENTS}

\section{A. The microradian x-ray diffraction}

The microradian diffraction of synchrotron radiation was demonstrated to be very efficient to characterize the ordering of the artificial opals. To study the IOLCs we used the same microradian setup as in $[25,26]$ at the Dutch-Belgian bending magnet BM26 DUBBLE beamline [27] at the European Synchrotron Radiation Facility (ESRF, Grenoble, France). An $x$-ray beam with photon energy of $13 \mathrm{keV}$ (wavelength $\lambda=0.95 \AA$, bandpass $\Delta \lambda / \lambda=2 \times 10^{-4}$, size at the sample $0.5 \times 0.5 \mathrm{~mm}^{2}$ ) was focused with compound refractive lenses [28] onto the phosphor screen of a two-dimensional detector (Photonic Science CCD camera, $4000 \times 2700$ pixels of $9 \mu \mathrm{m}^{2}$ ) located at 8 meters behind the sample.

The geometry of the experiment was similar to the one described in Refs. [17,18]. Figure 2 displays the scheme of the

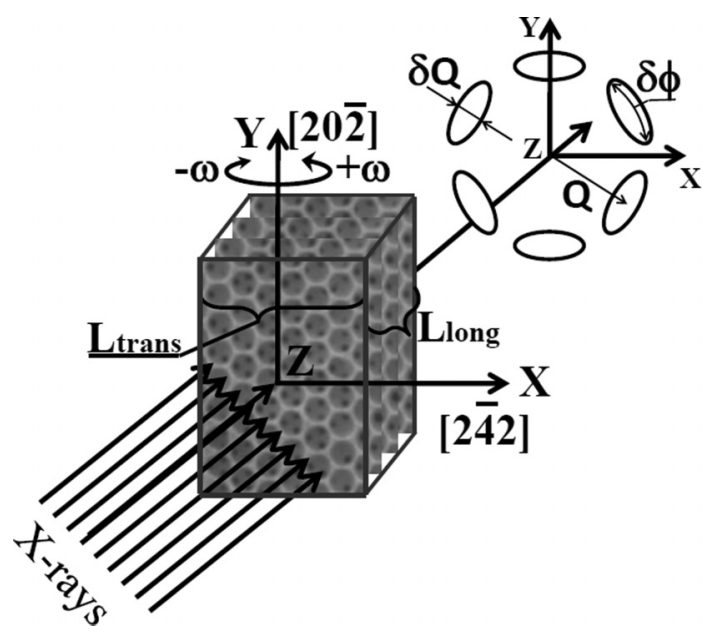

FIG. 2. Schematic drawing of the microradian x-ray diffraction experiment with the use of synchrotron radiation. 
experiment. The sample has been mounted on a goniometer, which allows precise orientation around three orthogonal axes to the sample surface. The opal-like films were first mounted perpendicular to the X-ray beam. Then the samples were rotated around the vertical axis $Y$ within the range $-75^{\circ}<\omega<75^{\circ}$, where $\omega$ denotes the angle of rotation; zero angle corresponds to the geometry of the sample surface being perpendicular to the beam axis. The diffraction patterns were recorded at each degree of rotation. A detailed description of the studied structural parameters is presented in the results section.

\section{B. The wide-angle $x$-ray diffraction}

The wide-angle (powder) $\mathrm{x}$-ray diffraction experiment was performed at the Swiss-Norwegian bending magnet BM01A beamline at the ESRF using the Pilatus2M detector. The wavelength was $\sim 0.7 \AA$, and the sample to detector distance was $144 \mathrm{~mm}$. The incident beam was kicked normal to the surface of the IOLC and transmitted providing a typical powder diffraction pattern, i.e., a system of concentric rings. The instrumental contribution to the peak broadening was determined with a $\mathrm{LaB}_{6}$ standard. A temperature-dependent diffraction experiments were performed in the temperature range of $80-500 \mathrm{~K}$ with the step of $\sim 1 \mathrm{~K}$.

All the obtained data were preprocessed with SNBL TOOL BOX [29] and azimuthally integrated with FIT2D [30]. In order to obtain temperature variation of the lattice parameter the Rietveld refinement was done for every temperature step (sequential refinement) with the FULLPROF software package [31]. To study atomic structure properties both the whole powder pattern modeling (WPPM) with PM2K software [32,33] and the Rietveld refinement with MAUD software [34] were carried out in order to confirm the validity of the obtained data.

\section{WILSON THEORY FOR THIN CRYSTALLINE FILMS WITH STACKING DISORDER}

For structures formed by monodisperse spherically symmetric objects the scattering intensity can be written as a product of the squared form factor $F^{2}(\mathbf{Q})$ representing the intraparticle scattering and the structure factor $S(\mathbf{Q})$, which describes interference between contributions originating from individual scattering objects $[12,35]$ :

$$
I(\mathbf{Q})=F^{2}(\mathbf{Q}) S(\mathbf{Q}),
$$

where $\mathbf{Q}$ is the diffraction wave vector (momentum transfer), which is defined by the difference of the wave vectors of incident and scattered beams.

For a uniform sphere of radius $R$ the form factor depends on the length of the diffraction wave vector $F(\mathbf{Q})=F(Q)$, and it equals

$$
F(Q)=\left|3 \frac{\sin (Q R)-(Q R) \cos (Q R)}{(Q R)^{3}}\right| .
$$

In contrast to the form factor describing scattering of a single particle, the structure factor $S(\mathbf{Q})$ depends on the position of the centers of the spheres, $\mathbf{r}_{\mathbf{i}}$ :

$$
S(\mathbf{Q})=\frac{1}{N}\left|\sum_{i=1}^{N} e^{i \mathbf{Q} \mathbf{r}_{\mathbf{i}}}\right|^{2}
$$

where $N$ denotes the number of particles.

Let us consider a layered structure formed by the hexagonal close-packed layers arbitrary stacked in the growth direction. For such a structure there are two types of reflections, the stacking-independent and stacking-dependent with the different structure factors [36,37]. If the sequence of layers is not perfect, i.e., possesses some deviations from ideal fcc or hcp, some additional features, namely diffuse Bragg rods passing through the stacking-dependent reflections, appear [36].

In Wilson theory the chance of finding a randomly chosen layer in the fcc environment is assumed to be given by a fixed stacking parameter $\alpha$. We denote $A_{n}$ the chance of finding an $n$th layer in the lateral position A. Consider a situation of adding layer $(n+2)$ to existing layers $n$ and $(n+1)$. This new layer can have the lateral position A in two cases, (i) if the structure is hcp and layer $n$ is also A, or (ii) if all three layers have different positions (BCA or CBA). Thus, one can define the recurrent relation between the probabilities of subsequent layers [12]:

$$
A_{n+2}=(1-\alpha) A_{n}+\alpha\left(1-A_{n}-A_{n+1}\right) .
$$

Without loss of generality one can assume that the 0th layer is in the A position; i.e., $A_{0}=1$. The probabilities can then be derived:

$$
A_{n}=\frac{1}{3}+H_{e} \rho_{e}^{n}+H_{0} \rho_{0}^{n},
$$

where

$$
\begin{aligned}
& \rho_{e}^{n}=\left(\frac{-\alpha+\sqrt{4-8 \alpha+\alpha^{2}}}{2}\right)^{n}, \\
& \rho_{0}^{n}=\left(\frac{-\alpha-\sqrt{4-8 \alpha+\alpha^{2}}}{2}\right)^{n}, \\
& H_{e}=\frac{1+2 \rho_{0}}{3\left(\rho_{0}-\rho_{e}\right)}, \quad H_{0}=\frac{1+2 \rho_{e}}{3\left(\rho_{e}-\rho_{0}\right)} .
\end{aligned}
$$

The chance of finding $n$th layer in $\mathrm{B}$ or $\mathrm{C}$ position is simply $\left(1-A_{n}\right) / 2$. Within the usual Wilson theory [12] the probabilities (5) are then used to derive the structure factor assuming that the crystal has an infinite number of layers. The result $[12,14,36,38,39]$ proves that some of the reflections are stacking-independent and remain sharp for any values of $\alpha$. The other reflections smear out into diffuse Bragg rods along the normal to close-packed hexagonal planes. The structure factor profile along the diffuse Bragg rods then sensitively depends on the stacking parameter $\alpha$.

The vertical deposition technique yields crystalline colloidal films with a finite number of layers. We have therefore extended Wilson theory to thin crystals with stacking disorder. In the following we shall assume that the structure can be still described using the same stacking parameter $\alpha$ as for an infinite crystal. In practice this assumption means that within the irradiated part of the crystalline film one finds many different realizations of the stacking sequence due to the presence of different domains and the presence of in-plane stacking disorder [40,41].

For a thin crystal the stacking-independent reflections will broaden in the usual way in the direction $z$ along the normal to the crystal film. In the reciprocal space it is convenient to introduce the dimensionless coordinate $l$ along $z$ as 
$l=Q_{z} /\left(2 \pi / d_{0}\right)$, where $d_{0}=2 R \sqrt{2 / 3}$. The structure factor profile $S(l)$ along $z$ for the stacking-independent reflections is then given by [37]

$$
S(l)=\frac{1}{N_{l}}\left(\frac{\sin \left(N_{l} \pi l\right)}{\sin (\pi l)}\right)^{2},
$$

where $N_{l}$ is the number of layers along the $z$ direction.

For the stacking-dependent reflection the summation over all statistically averaged realizations of a random-stacking structure yields [37]

$$
\begin{aligned}
S(l) \propto & \left\{-1+\frac{1}{N_{l}} 3 \operatorname{Re}\right. \\
& \times\left[H_{e} \frac{\left(\rho_{e} e^{i 2 \pi l}\right)^{N_{l}+1}-\left(N_{l}+1\right) \rho_{e} e^{i 2 \pi l}+N_{l}}{\left(1-\rho_{e} e^{i 2 \pi l}\right)^{2}}\right. \\
& \left.\left.+H_{0} \frac{\left(\rho_{0} e^{i 2 \pi l}\right)^{N_{l}+1}-\left(N_{l}+1\right) \rho_{0} e^{i 2 \pi l}+N_{l}}{\left(1-\rho_{0} e^{i 2 \pi l}\right)^{2}}\right]\right\} .
\end{aligned}
$$

Finally, one has to take into account that the form factor squared $F^{2}(Q)$ also varies along the Bragg rod due to variation of $Q$. The intensity profile can then be readily calculated using Eqs. (1), (2), and (7).

\section{ORDER AND DISORDER IN INVERSE OPAL-LIKE CRYSTALS}

\section{A. Mesoscopic structure}

In order to study the mesoscopic structure of Ni-based IOLCs the analysis of microradian X-ray diffraction patterns was carried out. The typical diffraction patterns of IOLCs are shown in Figs. 3(a)-3(c). The diffraction maps were recorded at different rotation angles $\omega=0^{\circ}(\mathrm{a}),-35^{\circ}(\mathrm{b})$, and $55^{\circ}(\mathrm{c})$. Figures 3(d)-3(f) present the expected Bragg reflections for an ideal fcc crystal with respective orientations. One can see that most of the Bragg peaks in Figs. 3(a)-3(c) correspond to a fcc structure.

(a)

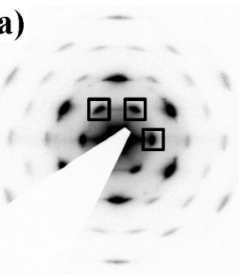

(b)
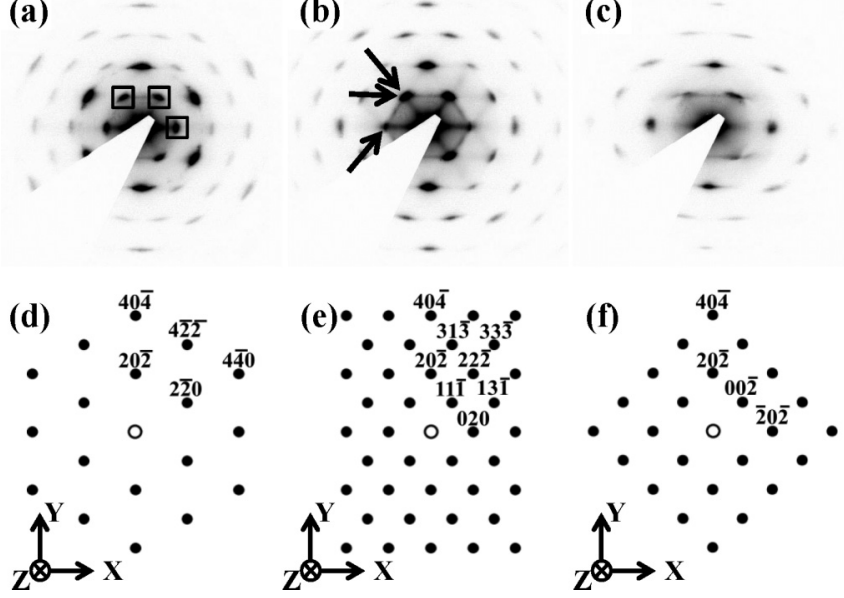

FIG. 3. (a)-(c) Microradian $\mathrm{x}$-ray diffraction patterns of $\mathrm{Ni}_{26}$. The patterns are measured at $\omega=0^{\circ}(\mathrm{a}),-35^{\circ}$ (b), $55^{\circ}$ (c). The sketches (d)-(f) illustrate reciprocal lattices for an ideal fcc crystal with the orientations corresponding to patterns (a)-(c).

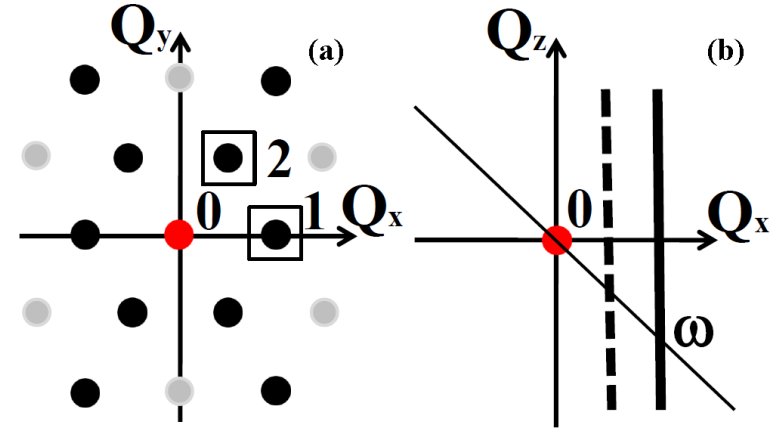

FIG. 4. (Color online) A sketch illustrating the Bragg rods orthogonal to the sample surface. (a) The top view of the reciprocal space (corresponding to the geometry of the sample surface being perpendicular to the $\mathrm{x}$-ray beam, $X Y$ plane). Numbers 1 and 2 denote the projections of two representative Bragg rods. Dark circles shows the projections of staking-dependent reflections. (b) The side view ( $X Z$ plane). The thick solid line is the Bragg rod 1; the dashed thick line is the Bragg rod 2. The latter is not in the $Q_{y}=0$ plane; that is why it is dashed. The inclined solid line shows the intersection of the Ewald sphere with reciprocal lattice points at a certain angle $\omega$.

Closer analysis of the pattern in Fig. 3(a) reveals some additional features shown with squares, which could correspond to 100 reflection of a hcp crystal formed by spheres of the same diameter. Their appearance can be understood with the help of Figs. 4(a) and 4(b), which show the projections of characteristic lattice points onto the sample surface $(X Y)$ plane and onto the $(X Z)$ plane, respectively. Indeed, the sample rotation reveals that the features correspond to diffuse Bragg rods along the [111] direction orthogonal to the $X Y$ plane. These rods pass through the stacking-dependent reflections, whose projections are marked in Fig. 4(a) with dark circles. According to our previous papers [5,11,17-20], these rods can be related either to the finite thickness of the colloidal crystal or to increased amount of planar defects and mechanical stress in the crystal. Apart from this, the paper [21] demonstrates surface truncation to be a probable reason for the appearance of the rods. Although for the samples under study the arrows in Fig. 3(b) show the rods along other $\langle 111\rangle$ directions of IOLCs, that is evidence of the presence of stacking faults, not only along the direction orthogonal to the sample surface (the substrate plane).

The experimental positions of the fcc peaks are in good agreement with the theoretical data; e.g., $Q_{(20 \overline{2})}^{\exp }=23.1 \pm 0.1 \mu \mathrm{m}^{-1} \quad$ and $\quad Q_{(20 \overline{2})}^{\text {cal }}=23.7 \mu \mathrm{m}^{-1}$, $Q_{(\overline{1} 1 \overline{1})}^{\exp }=14.1 \pm 0.1 \mu \mathrm{m}^{-1}$ and $Q_{(\overline{1} 1 \overline{1})}^{\text {cal }}=14.5 \mu \mathrm{m}^{-1}$ for polystyrene microspheres with the diameter of $530 \pm 10 \mathrm{~nm}$; i.e., the inaccuracy is $3 \%$. The lattice constant of these crystals is $a_{0}=750 \pm 10 \mathrm{~nm}$.

In order to carry out a detailed analysis of stacking disorder we evaluate an intensity profile along the Bragg rod in the [111] direction orthogonal to the sample surface, which corresponds to square marked in Fig. 4(a) by 1. Experimentally, when we turn the sample by an angle $\omega$, the Ewald sphere, which always passes through the origin of the reciprocal space marked by zero, will pass through the Bragg rod 1 at $Q_{z}=b_{1} \tan \omega$. Here $b_{1}=2 \pi /(R \sqrt{3})$ is the length of the in-plane basis vector of 


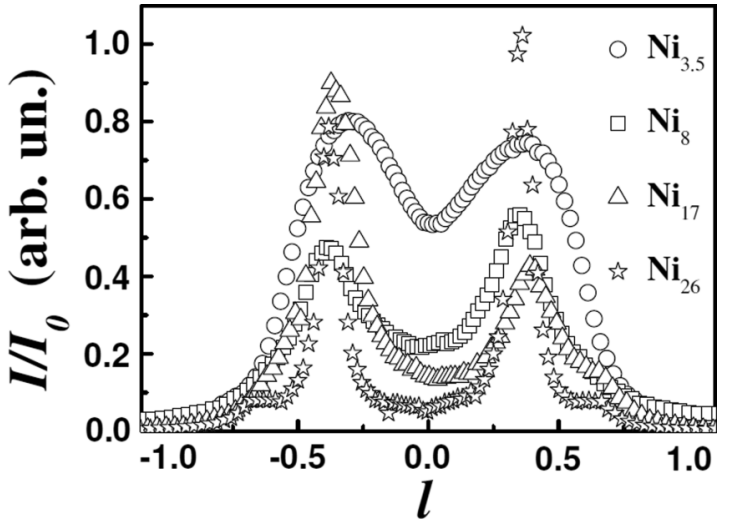

FIG. 5. The profiles of the normalized intensity distribution along Bragg rods 1 (see Fig. 4 for the definition).

the reciprocal lattice. Taking into account the definition of the dimensionless coordinate $l$ given in the theoretical section, for the Bragg rod 1 one can readily obtain $l=(2 \sqrt{2} / 3) \tan \omega$.

Figure 5 shows the obtained profiles of the normalized intensity distribution along the Bragg rods 1 . The presence of two symmetrically situated peaks in the profiles suggests that the crystals possess twinning planes. In other words, the crystals consist of domains with different stacking directions. The volume fractions of $A B C A B C \ldots$ and $A C B A C B \ldots$ twin components can be estimated from the ratio of integral intensities of these symmetrically located reflections [16]. The obtained ratio between the twin components does not demonstrate a clear dependence on the number of layers. So, the ratio is $1: 1$ for $\mathrm{Ni}_{3,5}$ samples, 4:5 for $\mathrm{Ni}_{8}$ and $\mathrm{Ni}_{26}$ samples, and 2:1 for the $\mathrm{Ni}_{17}$ sample as one can estimate from Fig. 5.

The quantitative analysis of structure imperfection was carried out taking into account that the number of hexagonal close-packed layers was known from the current transients [24] along with the SEM data. So, in order to find the value $\alpha$ modified Wilson theory adapted for thin samples was used [12,37]; see theoretical section for more details.

The results of scattering intensity distribution analysis along diffuse Bragg rods of IOLCs with different numbers of layers are shown in Fig. 6. Since the values of $\alpha$ are markedly higher than 0.5 the obtained IOLCs have dominant fcc packing.

The mosaicity of the IOLC film, i.e., disorientation of the domains within the sample plane ( $\delta \phi$; see Fig. 2$)$, was determined from the full width at half maximum (FWHM) of diffraction peaks in the azimuth direction in the patterns measured with the $\mathrm{x}$-ray beam orthogonal to the substrate. The average lateral domain sizes $L_{\text {trans }}$ (for transversal periodicity, across the synchrotron beam) were determined from averaged FWHM ( $\delta Q$ in Fig. 2$)$ of $20 \overline{2}$ and $\overline{1} 1 \overline{1}$ reflections. The average longitudinal domain sizes (for the periodicity along the beam) were estimated from the FWHM of the rocking curves $\Omega$ using the equation $L_{\text {long }}=2 \pi /\left(Q_{(20 \overline{2})}^{\exp } \sin \Omega\right)[18]$.

By analyzing Figs. 6(b), 6(c), and 6(d) one can make the following conclusions. First, the mosaicity $\delta \phi$ increases from $8^{\circ}$ to $10^{\circ}$ with the number of layers. Second, the average structural domain size $L_{\text {trans }}$ varies from 3.7 to $7.5 \mu \mathrm{m}$ without

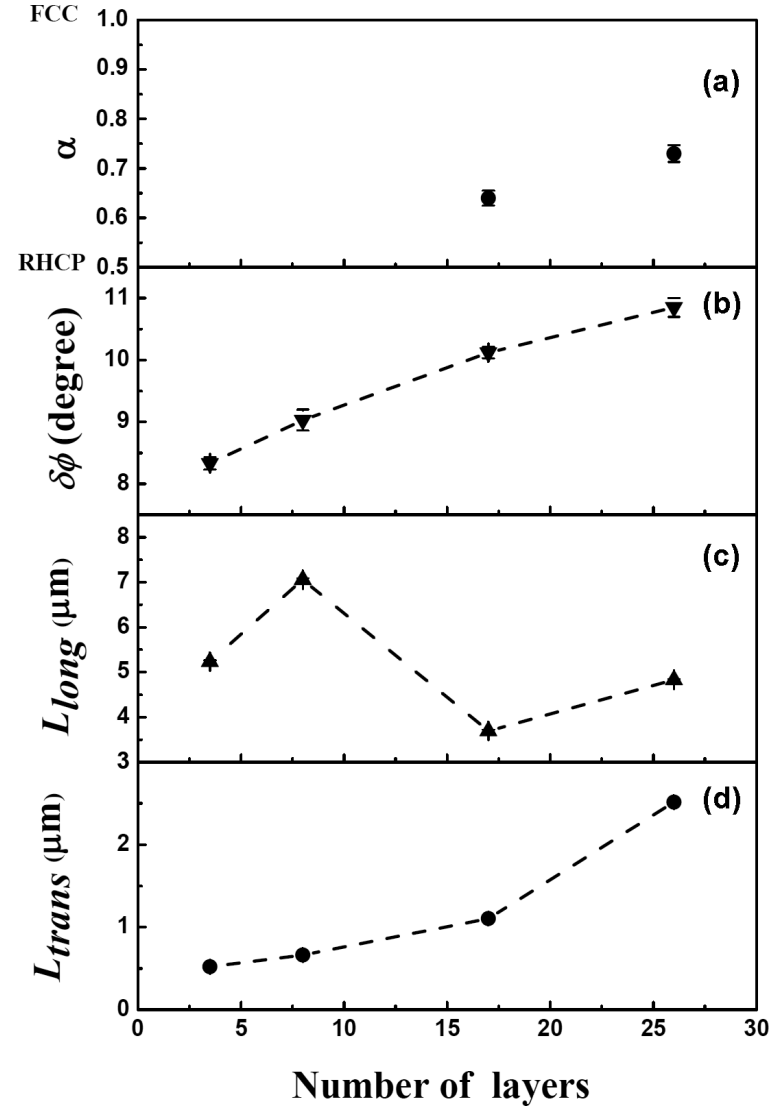

FIG. 6. Dependencies of IOLC main structural parameters according to microradian $\mathrm{x}$-ray diffraction data. (a) The probability of fcc motif formation $\alpha$, (b) the mosaicity $\delta \phi$, (c) the average size of structural domain along [202] direction $L_{\text {trans }}$, and (d) the average size of structural domain along [111] direction $L_{\text {long }}$.

clear dependence on the number of layers. Third, the average structural domain size along [111] direction $L_{\text {long }}$ increases from 0.5 to $2.8 \mu \mathrm{m}$ with the number of layers.

Thus the samples have got twinning fcc structure with average lateral domain size of about $5 \mu \mathrm{m}$. The domains are disoriented within an angle of $8^{\circ}-10^{\circ}$ in the sample plane. Taking into account that similar imperfections are peculiar for the original opal matrix [16,22], we can conclude that the IOLC structure mainly takes over the original matrix structure.

\section{B. Indirect method to study the layered structure of original matrices}

In general, the porous template for IOLC represents a thick colloidal crystal with at least 25-30 layers of polystyrene microspheres. It is plausible that its defect structure varies with the depth, i.e., with the distance from the substrate. To the best of our knowledge, there is no technique to study this structure on a large scale in a depth-resolved way. Even microradian $\mathrm{x}$-ray diffraction applied to the original colloidal matrix with several dozens of layers fails in this aspect, since it solely provides thickness-averaged information without any indication of thickness-resolved properties. However, using inverse crystals of variable thickness, one can learn about the depth-resolved structural properties of the original matrix 


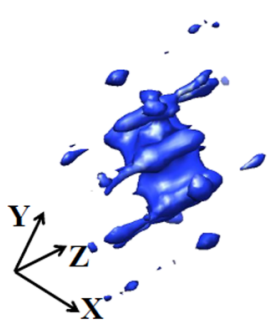

(a)

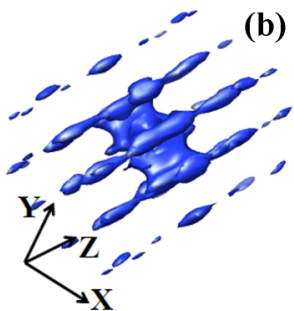

(b)

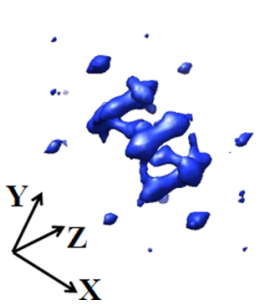

(c)

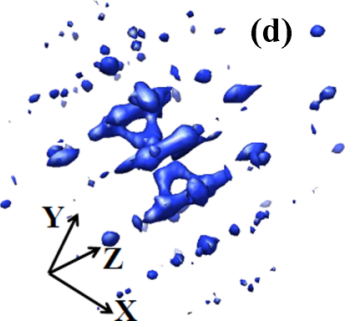

FIG. 7. (Color online) The 3D visualization of reciprocal space of inverse opal-like crystals (a) $\mathrm{Ni}_{3.5}$, (b) $\mathrm{Ni}_{8}$, (c) $\mathrm{Ni}_{17}$, and (d) $\mathrm{Ni}_{26}$. The numbers 1 and 2 relate to Bragg rods 1 and 2 introduced in Fig. 4.

assuming that the inversion process preserves the structure sufficiently well. To put it more exactly, our approach is based on microradian x-ray diffraction study and comparison of a number of IOLCs with different thickness, thereby implicitly characterizing the original matrix. Herewith, we propose using a $3 \mathrm{D}$ visualization of reciprocal space as a very intuitive representation $[42,43]$. For the reciprocal space reconstruction we used locally developed software.

Figure 7 presents 3D visualization of reciprocal space for the IOLCs. It is seen that all the samples possess the diffuse Bragg rods in the reciprocal space. These rods corresponds to hexagonal close-packed layers in the direct space located parallel to the substrate plane, i.e., perpendicular to the [111] direction. It is worth noting that only some of the reflections are elongated along the [111] direction, whose projections are marked in Fig. 4 by dark circles. This is characteristic for the layered structure with a considerable amount of stacking disorder [36]. This finding confirms the more significant impact of the stacking defects than effect of limited thickness of the sample.

The number of diffuse Bragg rods increases with the number of layers. When the number of layers reaches 8 , we can see rods which are aligned parallel to other $\langle 111\rangle$ directions [Fig. 7(b)]. This is additional evidence that stacking faults are also presented in other $\langle 111\rangle$ directions not only perpendicular to the sample plane. Nevertheless, the Bragg rods along the [111] direction are the most intense so that most stacking faults are parallel to the substrate. When the number of layers reaches 17 the diffuse Bragg rods still remain [Fig. 7(c)]; however the number of Bragg spots related to diffraction from other lattice planes increases. When the number of layers becomes above 25 , the number of reflection increases and the number of stacking faults decreases.

Thus by studying the dependence of the IOLC defect structure on the number of layers replicated from the initial opal-like matrix we can judge about thickness nonuniformity of the matrix itself. The obtained results tentatively suggest that the quality of the artificial opal crystals increases with the distance from the substrate. In other words, the region next to the substrate is more nonuniform.

\section{MICROSTRUCTURE OF THE NICKEL POLYCRYSTALLINE FRAMEWORK}

Wide-angle x-ray diffraction patterns for all the studied IOLCs present, as expected, diffraction peaks from the Ni fcc crystal structure with the lattice parameter $a$ of about $3.52 \AA$ (Fig. 8), in agreement with the standard data [44]. In addition, the patterns inevitably comprise peaks from the Au layer, which is always present in the consequence of sample synthesis. No other Bragg reflections have been noted in all the data, thus indicating structural purity of the polycrystalline nickel.

The microstructure that assumes size and strain effects was studied by thorough analysis of powder x-ray diffraction patterns collected at room temperature. Since the expected crystalline size is close to $50 \mathrm{~nm}$, that is nearly the resolution limit of our diffraction experiment, taking into account the instrumental resolution function is of much importance. Also, different types of defects are likely to coexist in the samples, e.g., dislocations as well as stacking faults. The analysis with WPPM and PM2K software [34] together with Rietveld refinement with MAUD software [32,33] was applied for every sample. As a result, a rough estimation of microstrains, crystallite sizes, and stacking fault probabilities was performed and cross checked using two methods mentioned above.

The best parametrization of the experimental patterns was obtained using the Warren approach [45] assuming stacking faults of the deformation type at $\{111\}$ planes and the Wilkens model $[46,47]$ with the dislocation possessing a $\{111\}\langle 110\rangle$ slip system. The implementation of this combined microstructural model does improve the quality of the agreement between observed and calculated profiles given by the conventional goodness of fit (GoF) values [48]. Thus by refining parameters of the above microstructure we observed that the GoF lowers more than twice, from 3.37 to 1.14 . Herewith, an almost perfect

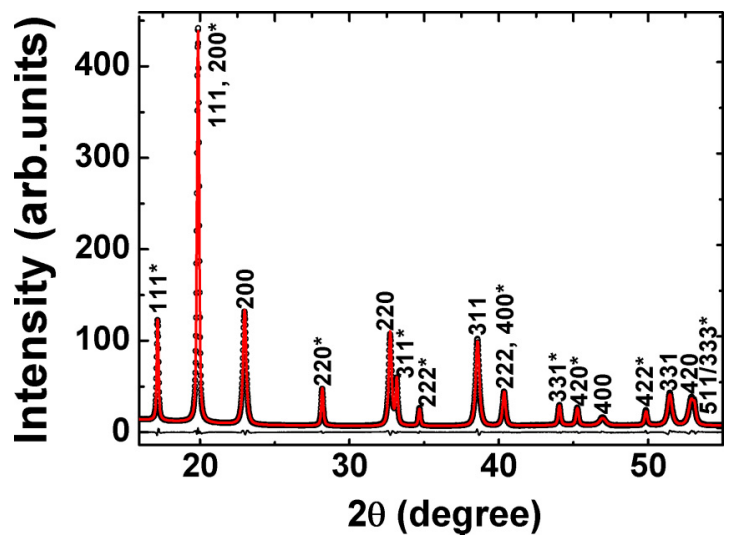

FIG. 8. (Color online) An example of the typical experimental $\mathrm{X}$-ray powder diffraction pattern with the profile obtained as a result of WPPM refinement and relative difference curve with reference to sample $\mathrm{Ni}_{17}$. The peaks are from $\mathrm{Ni}$ and $\mathrm{Au}$; the latter are marked with asterisks. 
matching to the experimental pattern was achieved as it is clearly seen in Fig. 8.

The obtained microstructural parameters coincide in the order of magnitude for all the studied samples. The size of the coherent scattering region is $\sim 50 \mathrm{~nm}$, being a good lower-bound estimate of average crystallite aggregates size, which is $\sim 100 \mathrm{~nm}$ according to the SEM data. The IOLC samples contain dislocations with the density higher than $1 \times 10^{15} \mathrm{~m}^{-2}$ as well as stacking faults of the atomic structure. The dislocation density is high but in a reasonable agreement with the values reported for powders and nanocrystalline thin films $[33,49]$. The concentration of twin stacking faults $(\sim 0.01)$ is higher than that for deformation-type stacking faults $(0.003 \pm 0.002)$, that is in agreement with the results of $[33,50]$.

The unit cell dimensions extracted from powder diffraction patterns were further used to characterize the response of the porous $\mathrm{Ni}$ polycrystal on the temperature variation. Following the authors of [51] the dependence of the lattice parameter on temperature $T$ was fitted in terms of the Debye model:

$$
a(T)=a_{0}+a_{0} C \Theta_{D} f\left(\Theta_{D} / T\right),
$$

where

$$
f(x)=3 \int_{0}^{1} \frac{t^{3}}{e^{t x}-1} d t,
$$

$\Theta_{D}$ is the Debye temperature, $a_{0}$ is the unit cell dimension approximated to $0 \mathrm{~K}$, and $C$ is a prefactor. The temperature evolution of the lattice parameter is exemplified for $\mathrm{Ni}_{17}$ in Fig. 9, which presents a good quality of the modeling at all the temperatures.

The results of the refinement show that the samples do not differ within experimental accuracy in terms of the Debye temperature. To give an example, for $\mathrm{Ni}_{17} \Theta_{D}=458 \pm 4 \mathrm{~K}$, which also in a reasonable agreement with the literature values as for single crystals and for powder [52,53]. This observation indicates that the low-frequency phonons in $\mathrm{Ni}$ are not affected by the mesoscopic structure.

The thermal expansion coefficient $C T E$ which corresponds to the first derivative of Eq. (8) divided by the lattice parameter at that temperature was averaged over the same temperature

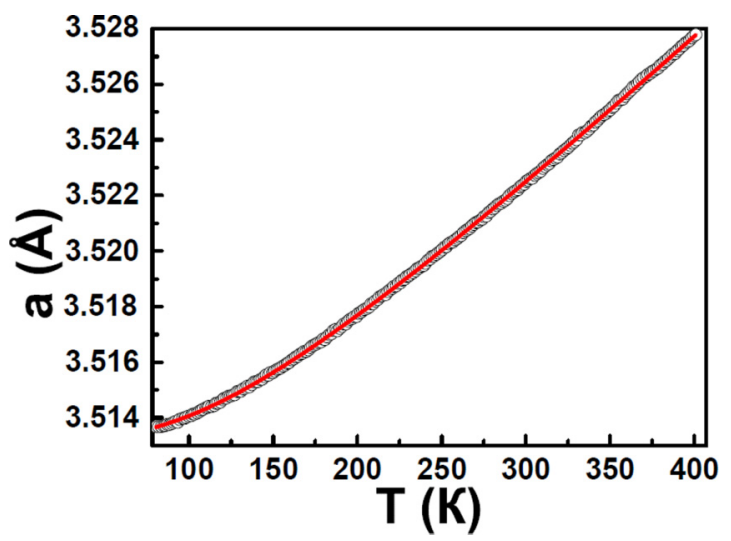

FIG. 9. (Color online) The typical temperature dependence of the lattice parameter with reference to sample $\mathrm{Ni}_{17}$. range as in Ref. [54], i.e., 173-298 K. In particular, the obtained $C T E$ value for the samples with a large number of layers $\left(\mathrm{Ni}_{17}\right.$ and $\left.\mathrm{Ni}_{26}\right)$ is $(1.33 \pm 0.05) \times 10^{-5} \mathrm{~K}^{-1}$, which agrees with the value for Ni thin film presented in Ref. [54] $((1.37 \pm 0.04) \times$ $\left.10^{-5} \mathrm{~K}^{-1}\right)$ and higher than that for bulk Ni $\left(1.24 \times 10^{-5} \mathrm{~K}^{-1}\right)$. According to Ref. [54] a higher value of CTE indicates smaller crystallite size, probably on the order of or less than $50 \mathrm{~nm}$ for thick samples, which is in a good agreement with our above-mentioned results based on powder diffraction patterns refinement.

As a result, the microstructural investigations show that, in fact, the walls of the IOLC structure are not fully continuous; rather their microstructural properties are similar to those for polycrystalline Ni film.

\section{CONCLUDING REMARKS}

In conclusion, the structure of metal inverse opals based on nickel has been studied on the mesoscopic and microscopic levels by SEM, microradian x-ray diffraction, and wide-angle $\mathrm{x}$-ray diffraction techniques.

The microradian $\mathrm{x}$-ray diffraction study resulted in the following conclusions. (i) The mesoscopic structure of IOLC based on $\mathrm{Ni}$ completely repeats the structure of the original matrix of the artificial opal crystal with minor alteration of the lattice constant. The formed structure is primary fcc (the probability of fcc motif formation, $\alpha \sim 0.7$ ) and its lattice constant is equal to $750 \pm 10 \mathrm{~nm}$. (ii) The additional features observed in the diffraction pattern are the diffuse Bragg rods, which are clearly seen by $3 \mathrm{D}$ visualization of the reciprocal space. These diffuse Bragg rods are situated not only along [111], which corresponds to substrate normal, but also along other $\langle 111\rangle$ directions in the IOLC, the [111] direction being the dominant one. These findings suggest the presence of the stacking faults, not only along the direction orthogonal to the sample surface. (iii) Investigation of a number of samples with different numbers of layers enabled us to reveal structural property variation with the thickness of the IOLCs. The average structure lateral domain size being about $5 \mu \mathrm{m}$ does not depend on the number of layers and it remains constant provided that original matrix is the same for all the samples. However, when the number of layers increases above 25 then the number of reflections increases and the number of stacking faults decreases. (iv) We proposed an indirect way to thickness-resolved study of the original artificial opal matrices, which is based on $3 \mathrm{D}$ visualization of the reciprocal space of IOLCs produced by the templated electrodeposition. As a result, we tentatively conclude that the quality of original templates increases with the distance from the substrate.

The results of wide-angle x-ray diffraction study can be summarized as follows. The IOLC samples contain Ni possessing fcc crystal structure with the lattice parameter of about $3.52 \AA$. The Ni framework is not completely uniform, while it is made up of rather large $(\sim 50 \mathrm{~nm})$ crystallites possessing the peculiar polycrystalline $\mathrm{Ni}$ dislocations and stacking faults. The thermal properties of the IOLC samples also agree with those for electrodeposited $\mathrm{Ni}$ thin film. 
Taken together, the above listed results show that the combination of two synchrotron-based experimental techniques, microradian diffraction and powder diffraction, provide detailed characterization on both average ordered structures and local disorder for both mesoscopic structure and atomic crystal structure of the framework material. IOLCs prepared with the templated electrodeposition mainly take over the original matrix structure, though the walls of the Ni framework are not fully continuous that should be accounted for in further magnetization distribution modeling. Thus, the obtained characteristics together with microscopic observations and macroscopic properties should serve as an experimental background for our understanding the physical response of inverse opal-like crystals, particularly their magnetic behavior.

\section{ACKNOWLEDGMENTS}

The authors acknowledge DUBBLE and SNBL beamlines for their hospitality. The work is supported in part by the Russian Foundation for Basic Research (Projects No. 12-02-12066, No. 14-08-00838, and No. 14-22-01113). In the part concerning the optimization of $\mathrm{Ni}$ electrodeposition conditions in porous templates the work is supported by the Russian Scientific Foundation (Grant No. 14-13-00809). We appreciate M. Leoni, D. Yu. Chernyshov, and I. A. Kasatkin for valuable suggestions concerning wide-angle X-ray diffraction data analysis. The Interdisciplinary Resource Center of Nanotechnology of Saint Petersburg State University is acknowledged for the SEM measurements. We also appreciate A. Bosak for providing his locally developed software for the $3 \mathrm{D}$ visualization of the reciprocal space.
[1] H. Yan, C. F. Blanford, B. T. Holland, M. Parent, W. H. Smyrl, and A. Stein, Adv. Mater. 11, 1003 (1999).

[2] L. Xu, W. L. Zhou, C. Frommen, R. H. Baughman, A. A. Zakhidov, L. Malkinski, J.-Q. Wang, and J. B. Wiley, Chem. Commun. 12, 997 (2000).

[3] P. N. Bartlett, P. R. Birkin, and M. A. Ghanem, Chem. Commun. 17, 1671 (2000).

[4] P. N. Bartlett, M. A. Ghanem, I. S. El Hallag, P. de Groot, and A. Zhukov, J. Mater. Chem. 13, 2596 (2003).

[5] K. S. Napolskii, A. Sinitskii, S. V. Grigoriev, N. A. Grigorieva, H. Eckerlebe, A. A. Eliseev, A. V. Lukashin, and Yu. D. Tretyakov, Physica B 397, 23 (2007).

[6] X. Yu, Y. J. Lee, R. Furstenberg, J. O. White, and P. V. Braun, Adv. Mater. 19, 1689 (2007).

[7] A. A. Grunin, N. A. Sapoletova, K. S. Napolskii, A. A. Eliseev, and A. A. Fedyanin, J. Appl. Phys. 111, 07A948 (2012).

[8] R. F. Wang, C. Nisoli, R. S. Freitas, J. Li, W. McConville, B. J. Cooley, M. S. Lund, N. Samarth, C. Leighton, V. H. Crespi, and P. Schiffer, Nature (London) 439, 303 (2006).

[9] M. N. Baibich, J. M. Broto, A. Fert, F. Nguyen, Van Dau, F. Petroff, P. Etienne, G. Creuzet, A. Friederich, and J. Chazelas, Phys. Rev. Lett. 61, 2472 (1988).

[10] P. Grunberg, R. Schreiber, Y. Pang, M. B. Brodsky, and H. Sowers, Phys. Rev. Lett. 57, 2442 (1986).

[11] A. A. Mistonov, N. A. Grigoryeva, A. V. Chumakova, H. Eckerlebe, N. A. Sapoletova, K. S. Napolskii, A. A. Eliseev, D. Menzel, and S. V. Grigoriev, Phys. Rev. B 87, 220408 (2013).

[12] A. J. C. Wilson, X-Ray Optics (Methuen \& Co. Ltd., London, 1949).

[13] J. Zhu et al., Nature (London) 387, 883 (1997).

[14] Ch. Dux and H. Versmold, Phys. Rev. Lett. 78, 1811 (1997).

[15] W. K. Kegel and J. K. G. Dhont, J. Chem. Phys. 112, 3431 (2000).

[16] J. Hilhorst, V. V. Abramova, A. Sinitskii et al., Langmuir 25, 10408 (2009).

[17] S. V. Grigoriev, K. S. Napolskii, N. A. Grigoryeva, A. V. Vasilieva et al., Phys. Rev. B 79, 045123 (2009).

[18] A. V. Vasilieva, N. A. Grigoryeva, A. A. Mistonov, N. A. Sapoletova et al., J. Physics: Conf. Ser. 247, 012029 (2010).

[19] N. A. Grigoryeva, A. A. Mistonov, K. S. Napolskii, N. A. Sapoletova et al., Phys. Rev. B 84, 064405 (2011).
[20] A. Sinitskii, V. Abramova, N. Grigorieva, S. Grigoriev, A. Snigirev, D. V. Byelov, and A. V. Petukhov, Europhys. Lett. 89, 14002 (2010).

[21] F. Marlow, M. Muldarisnur, P. Sharifi, and H. Zabel, Phys. Rev. B 84, 073401 (2011).

[22] K. S. Napolskii, N. A. Sapoletova, D. F. Gorozhankin et al., Langmuir 26, 2346 (2010).

[23] S.-L. Kuai, X.-F. Hu, A. Hache, and V.-V. Truong, J. Cryst. Growth 267, 317 (2004).

[24] N. Sapoletova, T. Makarevich, K. Napolskii, E. Mishina, A. Eliseev, A. van Etteger, T. Rasing, and G. Tsirlina, Phys. Chem. Chem. Phys. 12, 15414 (2010).

[25] A. V. Petukhov, J. H. J. Thijssen, D. C. 't Hart, A. Imhof, A. van Blaaderen, I. P. Dolbnya, A. Snigirev, A. Moussaid, and I. Snigireva, J. Appl. Cryst. 39, 137 (2006).

[26] J. Thijssen, A. Petukhov, D. C. 't Hart, A. Imhof, C. van der Werf, R. Schropp, and A. van Blaaderen, Adv. Mater. 18, 1662 (2006).

[27] M. Borsboom, W. Bras, I. Cerjak, D. Detollenaere, D. G. van Loon, P. Goedtkindt, M. Konijnenburg, P. Lassing, Y. K. Levine, B. Munneke, M. Oversluizen, R. van Tol, and E. Vlieg, J. Synchrotron Radiat. 5, 518 (1998).

[28] A. Snigirev, V. Kohn, I. Snigireva, and B. Lengeler, Nature (London) 384, 49 (1996).

[29] V. A. Dyadkin, SNBL Tool Box, Swiss Norwegian Beam Lines at ESRF, Grenoble, France, Release 2013-1

[30] A. P. Hammersley, FIT2D: An Introduction and Overview, ESRF Internal Report, 1997.

[31] J. Rodriguez-Carvajal, Physica B 192, 55 (1993).

[32] M. Leoni, T. Confente, and P. Scardi, Z. Kristallogr. Suppl. 23, 249 (2006).

[33] P. Scardi and M. Leoni, Acta Crystallogr. Sect. A: Found. Crystallogr. A58, 190 (2002).

[34] L. Lutterotti, Nucl. Instrum. Methods Phys. Res., Sect. B 268, 334 (2010).

[35] N. W. Ashcroft and N. D. Mermin, Solid State Physics (Saunders College Publishing, Philadelphia, 1976).

[36] A. V. Petukhov, I. P. Dolbnya, D. G. A. L. Aarts, G. J. Vroege, and H. N. W. Lekkerkerker, Phys. Rev. Lett. 90, 028304 (2003).

[37] X. Xian, M.Sc. thesis, van 't Hoff Laboratory for Physical and Colloid Chemistry, Utrecht University, 2003. 
[38] I. P. Dolbnya, A. V. Petukhov, D. G. A. L. Aarts, G. J. Vroege, and H. N. W. Lekkerkerker, Europhys. Lett. 72, 962 (2005).

[39] A. V. Petukhov, D. G. A. L. Aarts, I. P. Dolbnya, E. H. A. de Hoog, K. Kassapidou, G. J. Vroege, W. Bras, and H. N. W. Lekkerkerker, Phys. Rev. Lett. 88, 208301 (2002).

[40] J.-M. Meijer, V. W. A. de Villeneuve, and A. V. Petukhov, Langmuir 23, 3554 (2007).

[41] P. S. Miedema, V. W. A. de Villeneuve, and A. V. Petukhov, Phys. Rev. E 77, 010401 (2008).

[42] A. A. Eliseev, D. F. Gorozhankin, K. S. Napolskii, A. V. Petukhov, N. A. Sapoletova et al., JETP Lett. 90, 272 (2009).

[43] A. Bosak, I. Snigireva, K. S. Napolskii, and A. Snigirev, Adv. Mater. 22, 3256 (2010).

[44] H. E. Swanson and E. Tatge, U.S. Nat. Bur. Stds. Circular 539, 1 (1953).

[45] B. E. Warren, X-Ray Diffraction (Addison-Wesley, Reading, MA, 1969).
[46] M. Wilkens, Phys. Status Solidi A 2, 359 (1970).

[47] M. Leoni, J. Martinez-Garcia, and P. Scardi, J. Appl. Crystallogr. 40, 719 (2007).

[48] L. B. McCusker, R. B. Von Dreele, D. E. Cox, D. Louer, and P. Scardi, J. Appl. Crystallogr. 32, 36 (1999).

[49] G. Csiszar, K. Pantleon, H. Alimadadi, G. Ribarik, and T. Ungar, J. Appl. Crystallogr. 45, 61 (2012).

[50] P. G. Sanders, A. B. Witney, J. R. Weertman, R. Z. Valiev, and R. W. Siegel, Mater. Sci. Eng., A 204, 7 (1995).

[51] C. Roder, S. Einfeldt, S. Figge, and D. Hommel, Phys. Rev. B 72, 085218 (2005).

[52] R. H. Wilson, E. F. Skelton, and J. L. Katz, Acta Crystallogr., Sect. A: Found. and Advances 21, 635 (1966).

[53] Y. Tanji, J. Phys. Soc. Jpn. 30, 133 (1971).

[54] Y. Kuru, M. Wohlschlogel, U. Welzel, and E. J. Mittemeijer, Appl. Phys. Lett. 90, 243113 (2007). 